\title{
Knockdown of Long Non-Coding RNA XIST Inhibited Doxorubicin Resistanc in Colorectal Cancer by Upregulation of 124 and Downregulation of SGK1
}

\author{
Jia Zhu ${ }^{a}$ Rui Zhang ${ }^{b}$ Dongxiang Yang ${ }^{c}$ Jibin Lib Xiaofei Ya \\ Wenya Li $^{\mathrm{b}}$ Xin Liu ${ }^{\mathrm{b}}$ Jianfeng Zhao ${ }^{\mathrm{b}}$ Wen Shang ${ }^{\mathrm{b}}$ \\ aDepartment of Endoscopy, Cancer Hospital of China Medical Uni \\ Institute, Shenyang, ${ }^{b}$ Department of Colorectal, Cancer Hospital of $C$ \\ Cancer Hospital \& Insititute, Shenyang, 'Departmen rthopedics, ffiliated Hospital of Liaoning \\ University of Traditional Chinese Medicine, Shenyang, ral Imaging ancer Hospital of China \\ Medical University, Liaoning Cancer Hospital \& Insitit \\ eer $\operatorname{Jin}^{\mathrm{b}}$
} rang, China \section{de} (

Key Words

LncRNA $・ \mathrm{XIST} \cdot \mathrm{miR}-124 \cdot \mathrm{S}$

Abstract

Background/Aims: Dox cancer (CRC). However, for cancer therap $-M$ ou, contribute to $c^{\prime}$ molecular me ar $\mathrm{CRC}$ to DQ Yet 11) m. in DOX-resistant CRC tissues and cells were detected by qRT-PCR or kinase

alysis. DOX sensitivity was assessed by detecting IC50 value of DOX, the protein of P-y protein (P-gp) and glutathione S-transferase- $\pi($ GST- $\pi$ ) and apoptosis. The op butween XIST, miR-124 and SGK1 were confirmed by luciferase reporter assay, RT- $\quad d$ western blot. Xenograft tumor assay was used to verify the role of XIST in DOX stance in CRC in vivo. Results: XIST expression was upregulated and miR-124 expression stance of CRC cells, as evidenced by the reduced IC 50 value of DOX, decreased P-gp and $\checkmark S T-\pi$ levels and enhanced apoptosis in XIST-silenced DOX-resistant CRC cells. Additionally, XIST positively regulated SGK1 expression by interacting with miR-124 in DOX-resistant CRC cells. miR-124 suppression strikingly reversed XIST-knockdown-mediated repression on DOX resistance in DOX-resistant CRC cells. Moreover, SGK1-depletion-elicited decrease of DOX resistance was greatly restored by XIST overexpression or miR-124 inhibition in DOX-resistant 


\section{Cellular Physiology

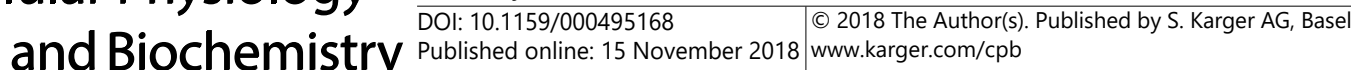 \\ Zhang et al.: XIST/miR-124/SGK1 Regulates DOX Resistance in CRC}

CRC cells. Furthermore, XIST knockdown enhanced the anti-tumor effect of DOX in CRC in vivo. Conclusion: XIST exerted regulatory function in resistance of DOX possibly through miR$124 /$ SGK1 axis, shedding new light on developing promising therapeutic strategy to overcome chemoresistance in CRC patients.

\section{Introduction}

Colorectal cancer (CRC) is one of the most frequently diagnosed cancers world and the third leading cause of cancer-associated mortality in the United St and females worldwide [1]. In recent years, the incidence and fatality rates of $\mathrm{C}$ an increasing trend in developed countries due to genetic and environ Chemotherapy is one of the important adjuvant therapy approaches particularly for those with advanced disease. Doxorubicin (DOX), an is widely used as a chemotherapeutic agent to fight with a broad including CRC [3]. However, the development of drug resistance [4]. Hen is imperatively needed to clarify the molecular mechanism of DOX res more effective intervention strategies for CRC.

Long noncoding RNAs (LncRNAs) are defined as evolu coding transcripts longer than 200 nucleotide 1 . It has bec oposed that IncRNAs play important roles in a series of physiological a thologica dcesses in tumorigenesis through affecting mRNA stability, RNA splicing tin structure, and miRNA-mediated gene regulation [6]. Moreover, ample evider nas rested that aberrantly expressed lncRNAs also contribute to drug resistance var tumors via different regulation mechanisms and signaling pathways [7- lactive specific transcript (XIST) is a product of XIST gene and a maste cg or lor X-chromosome inactivation in female mammals [8]. Dysregulation of XIST multiple neoplasms, highligb $\mathrm{p}$ and clinicopathological value in cancers [9]. Recent studies have docume that Xrorexpression was significantly increased in CRC tissues and cells, and fur ooncone in human CRC through facilitating cell proliferation, migration, asion a $\mathrm{a}[10,11]$. Moreover, it was also reported that XIST was significantly upregl d in refluorouracil (5FU)-resistant CRC cells, and enhanced XIST restrained 5FU-induced $\quad y$ in CRC cells by promoting the expression of thymidylate synthase [12]. $H^{\prime}$ whiner XIST participated in DOX resistance in CRC cells remains to be further ela

micro As a are a class of small, single-stranded ncRNAs of 20-22 nucleotides in lengt are al regulators of gene expression through binding to complementary s'aquen the 3 untranslated region (3'UTR) of mRNA [13]. Abundant studies have tha YAs, acting as potential oncogenes or tumor suppressors, are involved in the tion o. arug resistance in a variety of cancers [14], including CRC [15]. Recently, miRenriched miRNA, has been a focus of interest for its tumor-suppressive effect he acvelopment of many malignant tumors including CRC [16]. However, the function of
1ip 24 on chemoresistance in CRC has been scarcely understood till date. Recent studies est that IncRNAs can suppress the expressions and biological functions of miRNAs functioning as a molecular sponge or a competing endogenous RNA (ceRNA), hence leading to derepression of its target mRNA [17]. Interestingly, XIST was reported to directly interact with miR-124 to modulate bladder cancer progression [18]. Therefore, this study was designed to investigate whether XIST could interact with miR-124 to participate in the regulation of DOX resistance in CRC.

In the present study, we demonstrated the upregulation of XIST in DOX-resistant CRC cells. Functional analysis proved that XIST silencing reversed DOX resistance by facilitating 


\section{Cellular Physiology Cell Physiol Biochem 2018;51:113-128

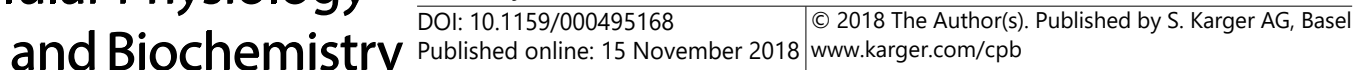

DOX-induced apoptosis in CRC cells. Mechanistic analyses revealed that XIST functioned as a molecular sponge to inhibit the expression and activities of miR-124, thus leading to increased expression of serum and glucocorticoid-inducible kinase 1 (SGK1) and enhanced resistance of DOX in CRC cells.

\section{Materials and Methods}

Patients and tissues

The study was approved by the Ethics Committee of Liaoning Cancer Hospital \& Insti informed consent was obtained from each patient prior to surgery. A total of 31 advanced CRC received 2 cycles of DOX-based adjuvant chemotherapy between 2014 and 2016 at Liao \& Institute were enrolled in this study. After completion of the chemotherapy, patien, chemosensitive group $(n=13)$ and chemoresistant group $(n=18)$ according to RECIS Criteria. All tumor specimens were obtained by surgical resection prior to underon

Cell lines and cell culture

Human CRC cell lines (HCT116 and LoVo) were obtained fro (ATCC, Manassas, VA, USA). All cells were maintained in RPMI-16 USA) supplemented with $10 \%$ heat-inactivated fetal bovine serum (F $\mathrm{mL}$ streptomycin (Invitrogen, Carlsbad, CA, USA), an incubator with a air flow of $5 \% \mathrm{CO}_{2}$ and $95 \% \mathrm{O}_{2}$ at $37^{\circ} \mathrm{C}$

The DOX-resistant CRC cells (HCT116/DOX and Loland LoVo cells through repeated exposure to incre $0.16,0.32$, and $0.50 \mu \mathrm{g} / \mathrm{mL}$ ) (Sigma, St. Louis, MO, US ver a -period. To maintain the DOX-resistant phenotype, HCT116/DOX and LoVo/DOX cells the culture medium containing $0.50 \mu \mathrm{g} /$ mL DOX.

Cell transfection

siRNA targeting XIST (si-XIs trol Sinva (si-Control), pcDNA empty control (pcDNA-Control), XIST-overexpressing plasmid o PNA- $\quad$-124 mimic (miR-124), control miRNA (miR-con), miR-124 inhibitor (anti-miR-124), ar IRNA in. or control (miR-Control) were purchased from GenePharma (Shanghai, China). Transien sfect $\$$ with these above plasmids or oligonucleotides into HCT116/DOX and LoVo/DOX cells yaner L Lipofectamine 2000 (Invitrogen, Carlsbad, CA, USA).

Lentivirus dy infection

Short pin 2 NA) targeting XIST (sh-XIST) were designed and synthesized by Genewiz, Inc. (Sur hina) serted into the pFH-L plasmid to generate lentiviral based shRNA expressing y tons. onfirmed by DNA sequencing, lentivirus vector carrying sh-XIST and packaging vectors -I an. $\mathrm{Y} \Delta \mathrm{R} 8.92)$ were cotransfected into HEK293T cells. The supernatant was collected at 48 nsfect 1 by centrifugation $\left(4000 \mathrm{~g}, 4^{\circ} \mathrm{C}, 10 \mathrm{~min}\right)$ and filtered through $0.45 \mu \mathrm{m}$ cellulose acetate tiviral vector that contained an irrelevant sequence was used as a negative control (lenti-

ontro1). For lentivirus infection, LoVo/DOX cells were seeded into 6-well plate at a density of $5 \times 10^{4}$ all yell and infected with lenti-sh-XIST or lenti-sh-Control at a multiplicity of infection (MOI) of 50 in resence of puromycin $(2 \mu \mathrm{g} / \mathrm{mL})$ and incubated for $72 \mathrm{~h}$ prior to performing the subsequent analyses. ne infection efficiency was determined through counting the number of infected green fluorescent protein (GFP)-expressing cells at 100× magnification under a fluorescence microscope (Olympus Corporation, Tokyo, Japan).

\section{RNA extraction and quantitative real-time ( $q R T-P C R)$ assay}

Total RNA was extracted from the collected tissues and cultured cells using TRIZOL regent (Invitrogen). $1 \mu \mathrm{g}$ of total RNA was reversely transcribed to complementary DNA (cDNA) in a final volume of $10 \mu \mathrm{l}$ using random primers and PrimeScript RT Reagent Kit (Invitrogen). Then the relative expression levels of XIST and miR-124 were performed using SYBR Premix Ex Taq (TaKaRa, Dalian, China) and TaqMan MicroRNA

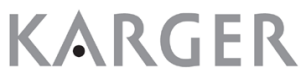




\section{Cellular Physiology Cell Physiol Biochem 2018;51:113-128 and Biochemistry \begin{tabular}{l|l} 
DOI: 10.1159/000495168 & (c) 2018 The Author(s). Published by S. Karger AG, Basel
\end{tabular} \\ Zhang et al.: XIST/miR-124/SGK1 Regulates DOX Resistance in CRC}

Assay Kit (Applied Biosystems, Foster City, CA, USA) on an ABI 7500 Fast Real-Time PCR system (Applied Biosystems), respectively. The qPCR reaction was set at $95^{\circ} \mathrm{C}$ for $10 \mathrm{~min}, 40$ cycles at $95^{\circ} \mathrm{C}$ for $15 \mathrm{~s}, 55^{\circ} \mathrm{C}$ for $30 \mathrm{~s}$, and $72^{\circ} \mathrm{C}$ for $30 \mathrm{~s}$. The primer sequences were displayed as below: XIST, $5^{\prime}$-ACGCTGCATGTGTCCTTAG-3' (forward) and 5'-GAGCCTCTTATAGCTGTTTG-3' (reverse); miR-124, 5'-GCTTAAGGCACGCGG-3' (forward) and 5'-GTGCAGGGTCCGAGG-3' (reverse); GAPDH, 5'-CATGAGAAGTATGACAACAGCCT-3' (forwar and 5'-AGTCCTTCCACGATACCAAAGT 3' (reverse); U6, 5'-CTCGCTTCGGCAGCACA-3' (forward) an 5'-AACGCTTCACGAATTTGCGT-3' (reverse). Expression levels of XIST and miR-124 were normalized to that of GAPDH and U6 small nuclear RNA (snRNA) using $2^{-\Delta \Delta C t}$ method.

Western blot

The cellular proteins were extracted from resected tissues or treated cells using the RIP (Beyotime, Beijing, China) supplemented with protease inhibitor cocktail (Sigma). Eq extractions $(50 \mu \mathrm{g})$ were subjected to $10 \%$ sodium dodecyl sulfate-polyacrylamide transferred to nitrocellulose membranes (Millipore, Billerica, MA, USA). After blocke for $2 \mathrm{~h}$ at room temperature, the membranes were then incubated at $4^{\circ} \mathrm{C}$ overnight wy against P-glycoprotein (P-gp, Santa Cruz Biotechnology, Santa Cruz, CA, USA), (GST- $\pi$ ) (Abcam, Cambridge, UK), SGK1 (Santa Cruz Biotechnology) actin (Sig, llowed by probed with horseradish peroxidase-conjugated secondary antibodies protein signals were detected by enhanced chemiluminescence sys (Bio-Rad, San Francisco, California, USA).

Drug resistance assay

3-(4, 5-dimethylthiazol-2-yl)-2, 5-diphenyltetrazo evaluate DOX sensitivity. Transfected HCT116/DOX (3000 cells/well) and incubated overnight. Cells were $0.1,0.5,1,5$, and $10 \mu \mathrm{g} / \mathrm{mL}$ ) for $24 \mathrm{~h}$. Subsequo 20
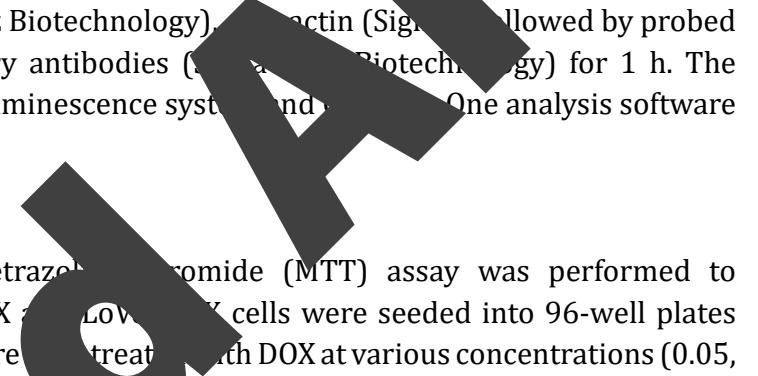
mide (MTT) assay was performed to Lon cells were seeded into 96-well plates solution ( $5 \mathrm{mg} / \mathrm{mL}$; Sigma) was added to each well and incubated for $4 \mathrm{~h}$, followed $b$ of curture medium and addition of $150 \mu \mathrm{l}$ dimethyl sulfoxide (DMSO; Sigma). The optical den reader (Bio-Rad Laboratories, In The half maximal inhibitory concentration (IC50) of DOX, at which drug produced bibition growth, was calculated according to the charted doseresponse curve at different cor

Flow cytometry analys

Transfected HCT116/D o/DOX cells were inoculated into 96-well plates and treated with Detection Kit (K Jiangsu, China). The apoptotic rate was measured by flow cytometry using a FACS flow Bme Be Dickinson, San Jose, CA, USA). (P) predicted miR-124 binding sites were amplified and IT2) and XIST-MUT (XIST-MUT1 or XIST-MUT2). Similarly, the predicted or mutated miR-124 ting sres in 3'UTR of SGK1 were amplified and cloned into pMIR-Report vector to generate (SGK1-WT) GK1-MUT). The mutant miR-124 target sites for XIST (XIST-MUT1 or XIST-MUT2) or SGK1-3'UTR as internal control pRL-TK (Promega) and miR-124 or miR-Control using Lipofectamine 2000 (Invitrogen). At $48 \mathrm{~h}$ posttransfection, cells were harvested and luciferase activity was measured using a Dual-Luciferase Reporter Assay System (Promega, Madison, WI, USA). The relative luciferase activity was normalized to Renilla luciferase activity.

Xenograft assay

The protocol was approved by the Animal Care and Ethics Committee of Liaoning Cancer Hospital \& Institute. All experimental procedures were performed in accordance with the National Institutes of Health 


\section{Cellular Physiology \begin{tabular}{ll|l} 
and BiOChemistry Published onIIne: I5 November 2018 & $\begin{array}{l}\text { (c) } 2018 \text { The Author(s). Published by S. Karger AG, Basel } \\
\text { www.karger.com/cpb }\end{array}$ \\
\hline
\end{tabular} \\ Zhang et al.: XIST/miR-124/SGK1 Regulates DOX Resistance in CRC}

Animal Use Guidelines. Female BALB/c nude mice (4-6 weeks old, n=5/group) were obtained from the Chinese Academy of Medical Sciences (Beijing, China) and housed under pathogen-free conditions. LoVo/ DOX cells stably transfected with lenti-sh-XIST or lenti-sh-Control were harvested and resuspended at a concentration of $1 \times 10^{8}$ cells $/ \mathrm{mL}$, and then $100 \mu \mathrm{L}$ of cell suspension was subcutaneously injected in a single side of the posterior flank of each mouse. Six days after cell injection, DOX was intraperitonea administered at a dose of $5 \mathrm{mg} / \mathrm{kg}$ every 3 days. Tumor growth was examined every 3 days, and tumo

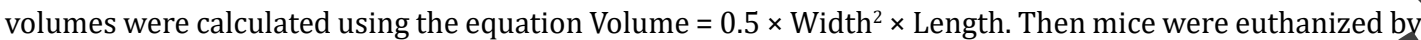
cervical dislocation at 21 days after treatment. Tumor masses were dissected, photographed and subjer to weight measurement, qRT-PCR analysis and western blot assay.

\section{Statistical analysis}

All data are presented as the means \pm standard deviation (SD). Statistical differ using one-way analysis of variance (ANOVA) or Student's $t$ test with SPSS version $18 \mathrm{~s}$ USA). Differences were considered significant when $P<0.05$.

\section{Results}

XIST was upregulated and miR124 was downregulated in DOXresistant CRC tissues and cells

To investigate the involvement of XIST and miR-124 in the DOX resistance of CRC, we initially checked the expression profiles of XIST and miR-124 in 31 CRC tumor tissues that were divided into chemosensitiv group ( $\mathrm{n}=13)$ and chemoresista group $(\mathrm{n}=18)$ by qRTresults showed that XIST e vion was robustly upregu eor 1A) and miR-124 ex osion distinctly downregulat (Fig. 1P) in tumor tissues from ch tant patients comp th trat from chemosensiti $p$ Then, the expression of ST ad miR-124 in the resis CRC cell lines (MCT1 $\mathrm{X}$ and LoVo/DOX) and pon arental CRC cell lines 16 an LoVo) were further eval y qRT-PCR. As expected, $T$ expression was apparently (Fig. 1C and 1D) and miR-124 ession was greatly lower (Fig. and $1 F$ ) in DOX-resistant CRC cell lines than that in their parental CRC cell lines. These results suggested that abnormal expressions of XIST and miR-124 may be associated with DOX resistance in CRC.

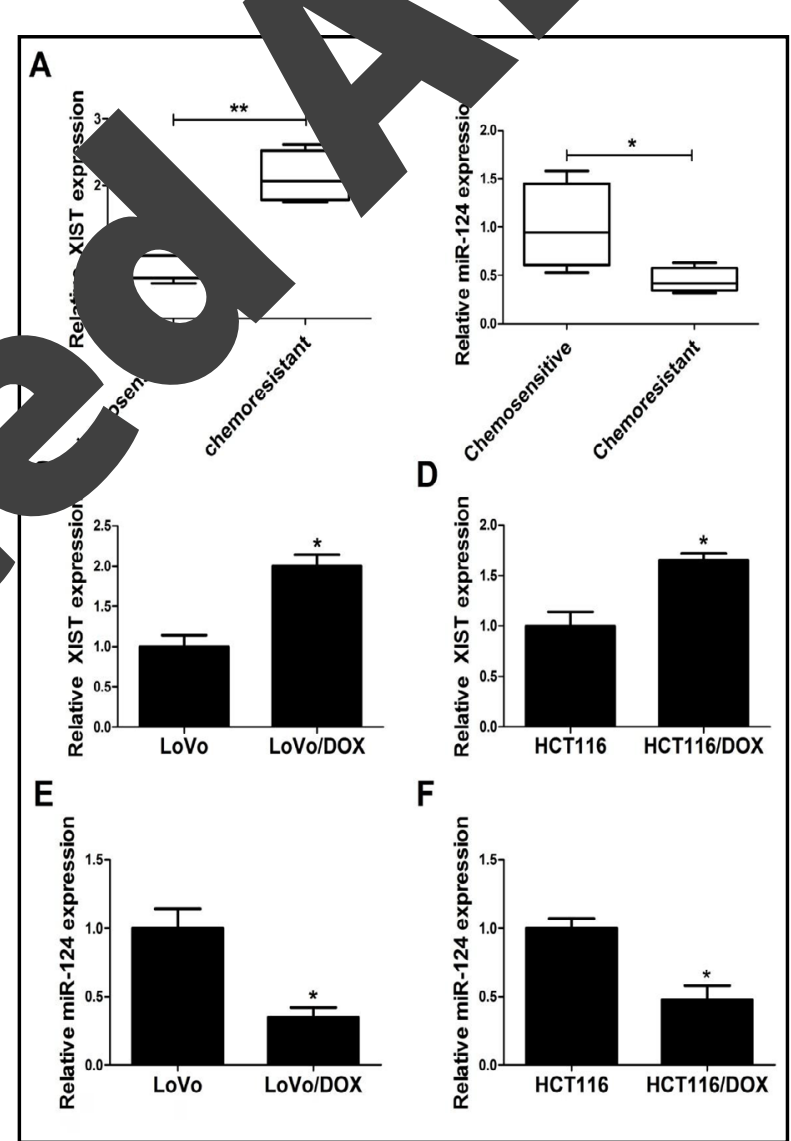

Fig. 1. XIST expression was increased and miR-124 expression was decreased in DOX-resistant CRC tissues and cells. qRT-PCR analyses of XIST (A) and miR-124 (B) expressions in 31 cases of CRC tumor tissues, among which there were 13 chemosensitive cases and 18 chemoresistant cases. qRT-PCR analyses of XIST (C and D) and miR-124 (E and F) in DOX-resistant CRC cell lines (HCT116/DOX and LoVo/DOX) and corresponding parental CRC cell lines (HCT116 and LoVo). ${ }^{*} \mathrm{P}<0.05$. 


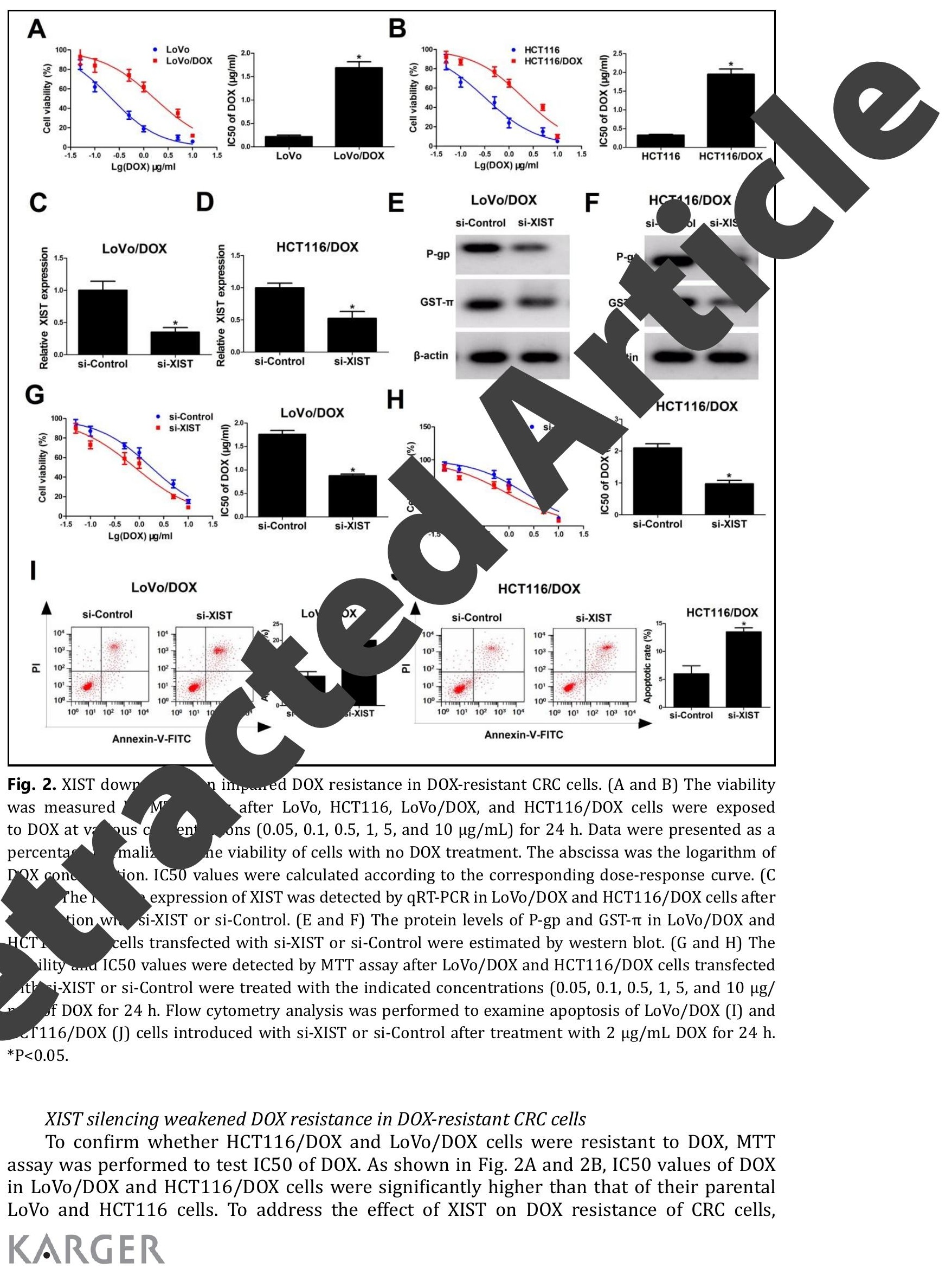




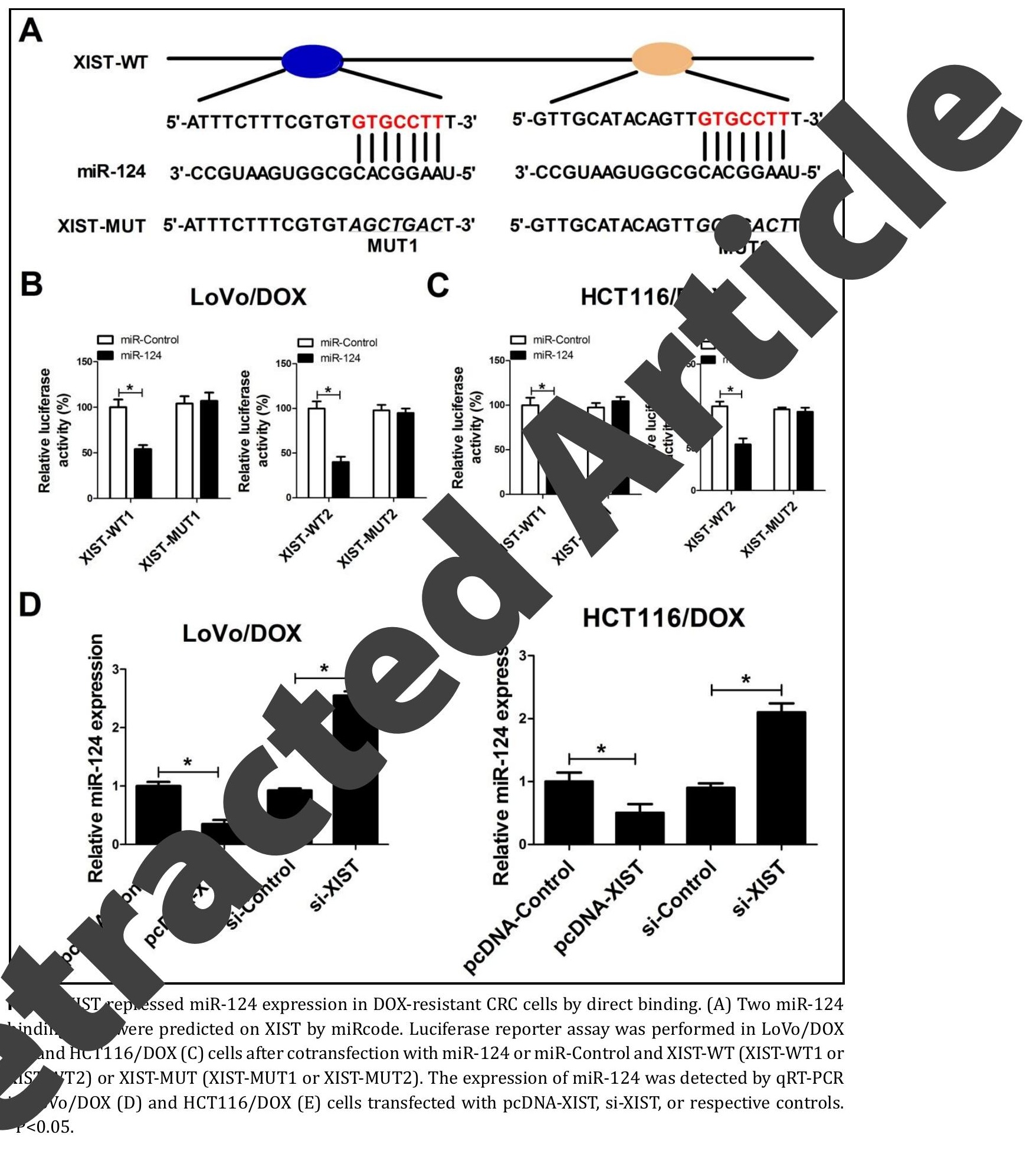

siRNA-mediated knockdown of XIST was performed in LoVo/DOX and HCT116/DOX cells by transfecting with si-XIST. As expected, XIST expression was greatly decreased in LoVo/ DOX (Fig. 2C) and HCT116/DOX (Fig. 2D) cells following transfection with si-XIST. It is wellknown that various transporter proteins including P-gp and GST- $\pi$ have been shown to be involved in chemotherapeutic resistance in cancers [19]. Western blot analysis revealed that XIST depletion led to a marked reduction of P-gp and GST- $\pi$ expressions in LoVo/DOX (Fig. 2E) and HCT116/DOX (Fig. 2F) cells. Consistently, MTT assay also implicated that si- 
Fig. 4. XIST depletion reduced chemoresistance of DOX-resistant CRC cells to DOX via regulating miR-124. LoVo/ DOX and HCT116/ DOX cells were transfected with miR-Control, miR124, si-Control, si-XIST, si-XIST + anti-miR-Control, or si-XIST + anti-miR-124. Western blot was performed to detect the protein levels of P-gp and GST- $\pi$ in transfected LoVo/ DOX (A) and HCT116/DOX (B) cells. MTT assay was employed to measure IC50 value of DOX in transfected LoVo/ DOX (C) and HCT116/DOX (D) cells after treated with different concentrations $(0.05,0.1,0.5,1$ 5 , and $10 \mu \mathrm{g} / \mathrm{m}$ of DOX for $h$. Flow try

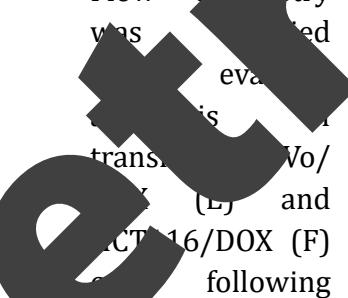

ated with $2 \mu \mathrm{g} /$ mL DOX for $24 \mathrm{~h}$. $* \mathrm{P}<0.05$.
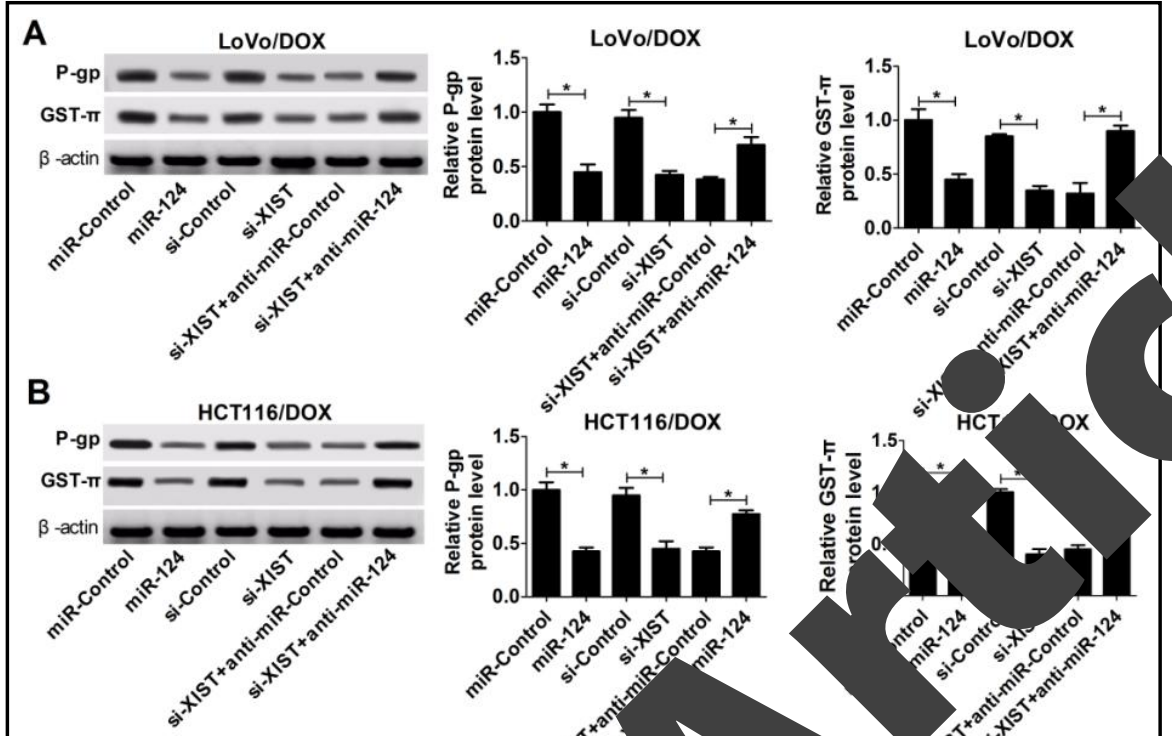

C
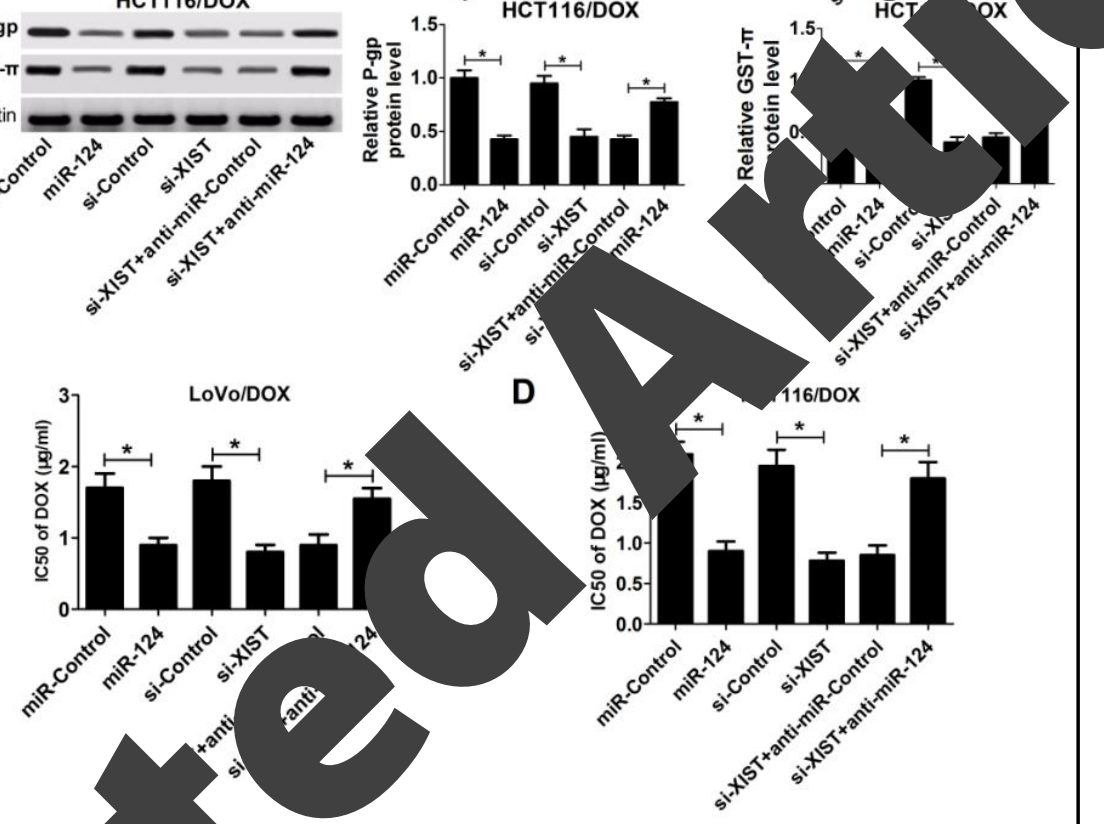

E
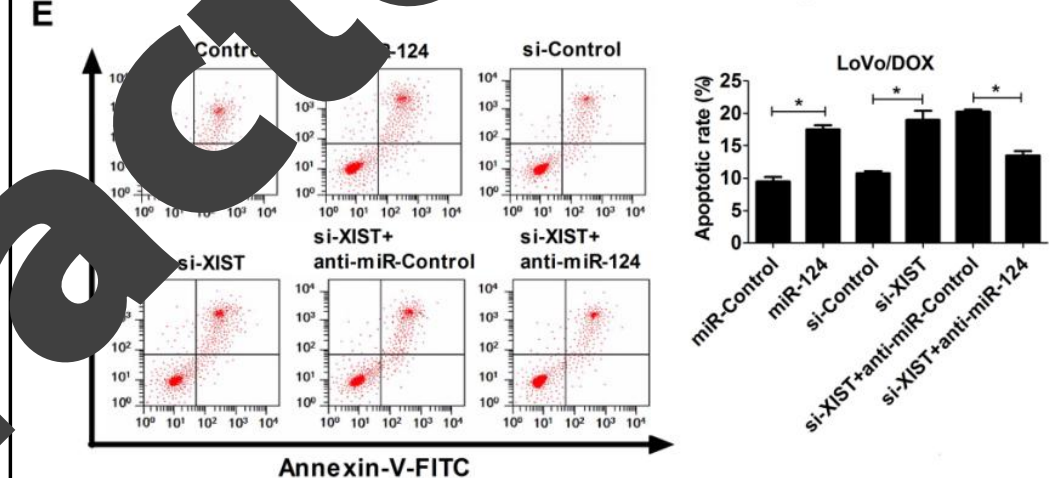

$F$
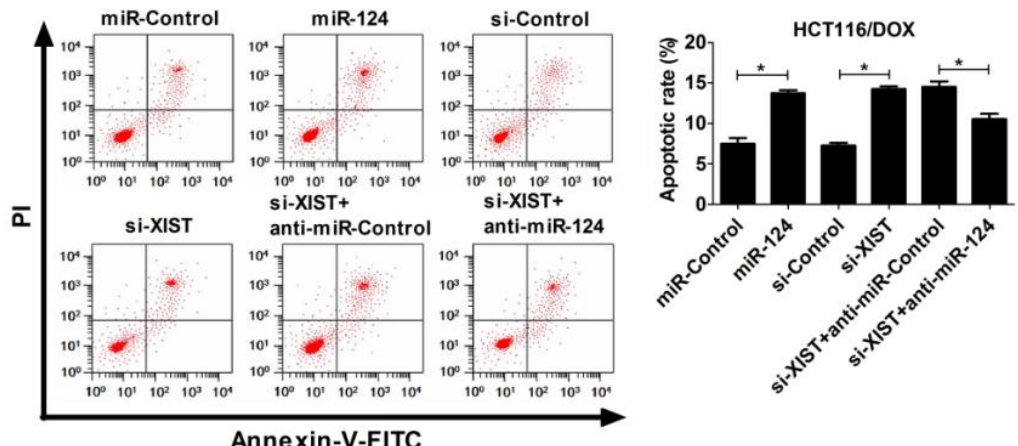


\section{Cellular Physiology and Biochemistry \begin{tabular}{l|l} 
DOI: $10.1159 / 000495168$ & $\begin{array}{l}\text { (c) } 2018 \text { The Author(s). Published by S. Karger AG, Basel } \\
\text { www.karger.com/cpb }\end{array}$
\end{tabular} \\ Zhang et al.: XIST/miR-124/SGK1 Regulates DOX Resistance in CRC}

XIST transfection in LoVo/DOX (Fig. 2G) and HCT116/DOX (Fig. 2H) cells rendered both cell lines more sensitive to DOX-mediated cytotoxicity compared with si-Control group, as demonstrated by the decreased IC50 value of DOX following XIST downregulation. Additionally, we further evaluated whether the effect of XIST on DOX resistance of CRC cells was associated with apoptosis. LoVo/DOX and HCT116/DOX cells transfected with Control or si-XIST were treated with $2 \mu \mathrm{g} / \mathrm{mL}$ DOX for $24 \mathrm{~h}$. Flow cytometry analysis resul demonstrated that XIST knockdown enhanced DOX-induced apoptosis in LoVo/DOX (Fig. 2I and HCT116/DOX (Fig. 2J) cells with respect to si-Control group. Collectively, these res indicated that XIST silencing enhanced DOX-induced cytotoxicity in DOX-resistant CRC

$X I S T$ regulated miR-124 expression in DOX-resistant CRC cells by direct interac

To elucidate the potential mechanism of XIST function in DOX-re two bioinformatic databases (starBase and TargetScanS) were employea arch h potential miRNAs that can be regulated by XIST. Bioinformatics tools an war two potential binding sequences of miR-124 on XIST gene (Fig. 3A). To can the action between XIST and miR-124, luciferase reporter constructs contain predicted wildtype or mutated miR-124 binding sites on XIST were g ciferase assays manifested that cotransfection with miR-124 mimic and prominently reduced the luciferase activity, while cotran tio giR-124 and XISTMUT (XIST-MUT1 or XIST-MUT2) had no obvious effect on ase ctivity in LoVo/DOX (Fig. 3B) and HCT116/DOX (Fig. 3C) cells. Next further mo red the expression of miR124 in response to the change XIST expression QX-resista $A$ RC cells. qRT-PCR results implied that miR-124 expression was specificall ted after overexpression of XIST and evidently promoted following knockdown of $X$ in oVo/DOX (Fig. 3D) and HCT116/ DOX (Fig. 3E) cells. Therefore, we concluded XI Wuld directly bind to miR-124 to suppress its expression in DOX-resistan

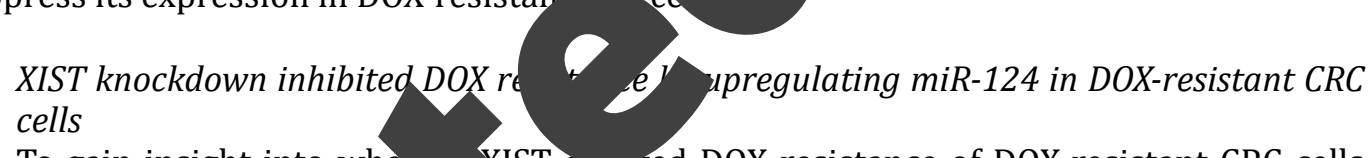

To gain insight into whe YIST arrected DOX resistance of DOX-resistant CRC cells via modulation of miR-12 nd HCT116/DOX cells were transfected with miR124 mimic, si-XIST or SIST+an $1 \mathrm{R}-124$. Western blot analysis demonstrated that ectopic expression of $r$ XIST knockdown distinctly reduced the protein levels of P-gp and GST-T-inLo rig. 4A) and HCT116/DOX (Fig. 4B) cells, while miR-124 antagomir mar versea the reduction of P-gp and GST- $\pi$ levels mediated by XIST silencing. MT2 SP that forced expression of miR-124 or downregulation of XIST greatly dir ish th 50 value of DOX in LoVo/DOX (Fig. 4C) and HCT116/DOX (Fig. 4D) cel nerea IST-induced decrease of IC50 was partially restored by miR-124 iv hibitio thermore, flow cytometry analysis indicated that miR-124 overexpression or nock dramatically aggravated DOX-induced apoptosis of LoVo/DOX (Fig. 4E) and 6/DOA,Fig. 4F) cells, however, si-XIST-triggered apoptosis was attenuated after co-

pression counteracted XIST-knockdown-mediated repression on DOX resistance in DOXant CRC cells.

XIST positively regulated SGK1 expression by interacting with miR-124 in DOX-resistant CRC cells

To address the underlying mechanism by which miR-124 exerted its effects on DOX resistance of CRC cells, bioinformatics tools (microRNA.org and miRBase) were used to search for the potential targets of miR-124. As displayed in Fig. 5A, binding sites of miR124 were identified in the 3'UTR of SGK1 mRNA. SGK1 has been reported to participate in cell survival, de-differentiation, cell cycle control, apoptosis, as well as chemo-and radio-resistance in various malignancies [20]. Moreover, a recent document points out that SGK1 is essential and rate limiting in the control of precursor miRNA nuclear export

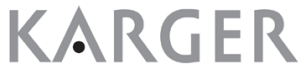


Fig. 5. XIST facilitated SGK1 expression via sponging miR-124 in DOX-resistant CRC cells. (A) The constructed luciferase reporter plasmids containing the wild-type or mutated miR-124 binding sites in the 3'UTR of SGK1. Luciferase reporter assay was performed to measure luciferase activity in LoVo/DOX (B) and HCT116/DOX (C) cells cotransfected with miR-124 or miRControl and SGK1-WT or SGK1-MUT. Western blot was performed to examine the protein level of SGK1 in LoVo/DOX (D) and HCT116/DOX (E) cells introduced with si-Control, si-XIST, miRControl, miR-124, miR$124+$ pcDNA-Control, or miR-124 + pcDNA-XIST. (F) qRT-PCR analysis of SGK1 mRNA expression in CRC tumor tissues (chemosensitive cases, $\mathrm{n}=13$; chemoresistant cases, $\mathrm{n}=18)$. ( $\mathrm{G}$ and $\mathrm{H}$ ) qRT-PCR analysis CRC cell lin LoVd OX

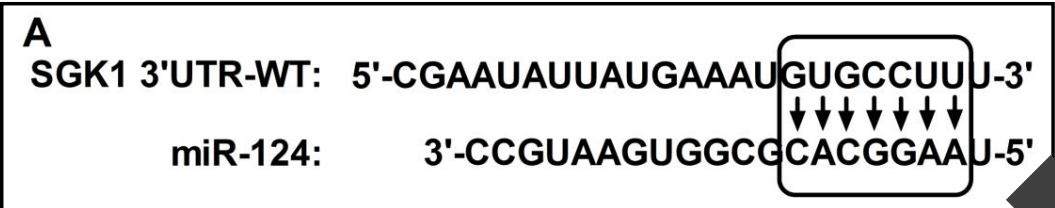

\section{SGK1 3'UTR-MUT: 5'-CGAAUAUUAUGAAAUGCAGACUU-3'}

B

C

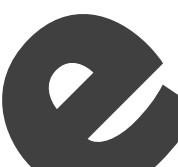

LoVo/DOX

HCT116/DOX

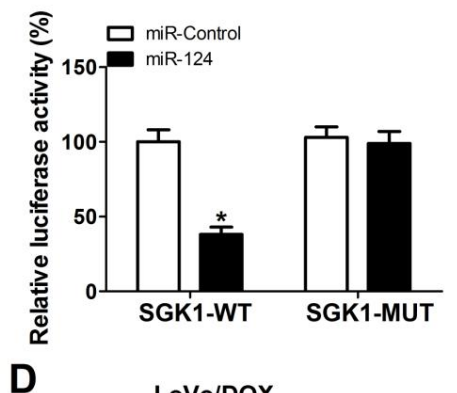

D LoVo/Dox
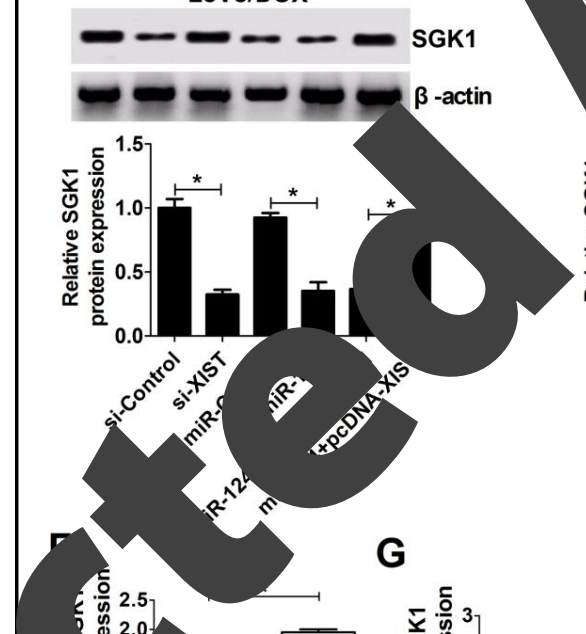

G
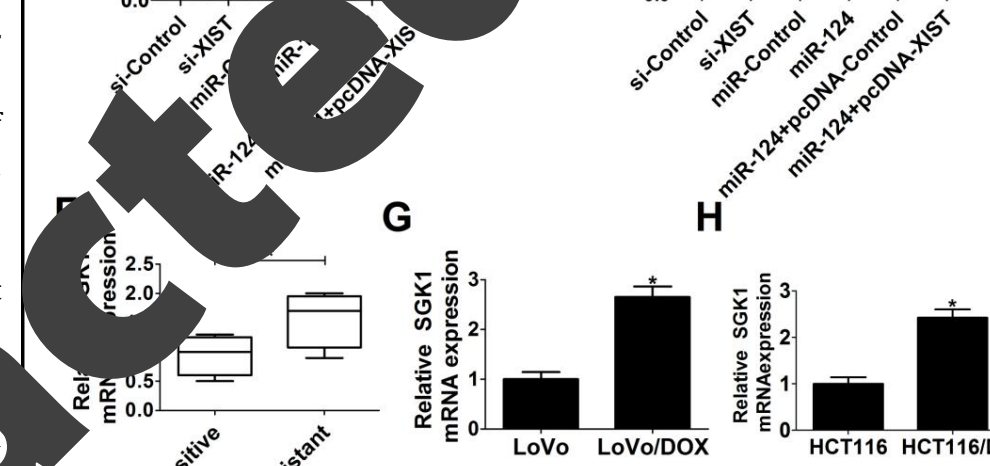
=
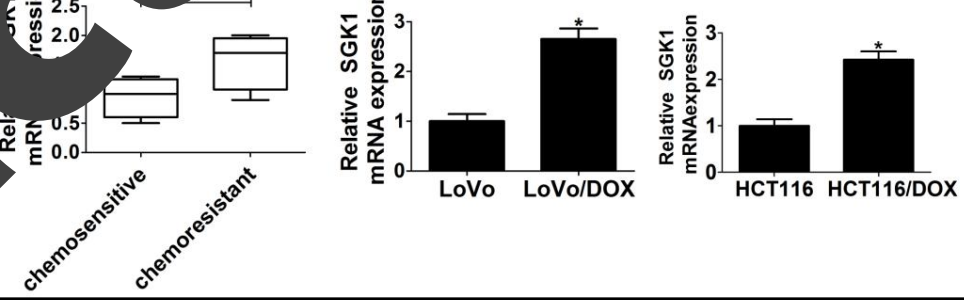
and $\mathrm{HCT}^{\mathrm{T}}$ parental

$$
\text { lin }{ }^{*} \mathrm{P}<0 \text {. }
$$

equent luciferase reporter assay revealed that miR-124 overexpression could ie tively suppress the luciferase activity of the reporter containing the wild-type miR-124 b. Ing sites in the 3'UTR of SGK1 in LoVo/DOX (Fig. 5B) and HCT116/DOX (Fig. 5C) cells, wever, these effects were disappeared when the binding sites were mutated. Furthermore, we found that XIST knockdown or miR-124 overexpression triggered a substantial decline of SGK1 protein level in LoVo/DOX (Fig. 5D) and HCT116/DOX (Fig. 5E) cells. Moreover, miR124-mediated decrease of SGK1 protein expression was significantly recuperated following XIST upregulation (Fig. 5D and 5E). All of these data made us draw a conclusion that XIST positively regulated SGK1 expression by interacting with miR-124 in DOX-resistant CRC cells. Additionally, as we might expect, SGK1 expression was increased in DOX-resistant CRC tumor tissues (Fig, 5F) and cells (Fig. 5G and 5H) when compared with respective controls. 
Fig. 6. SGK1-knockdownmediated decline of DOX resistance was regulated by XIST or miR-124 in DOXresistant CRC cells. LoVo/ DOX and HCT116/DOX cells were transfected with siSGK1, or combined with pcDNA-XIST, or anti-miR-124. Western blot was performed to detect the protein level of SGK1 in the transfected LoVo/DOX (A) and HCT116/ DOX (B) cells. MTT assay was used to measure the IC50 value of DOX in the transfected LoVo/DOX (C) and HCT116/DOX (D) cells after exposed to different concentrations $\quad(0.05,0.1$, $0.5,1,5$, and $10 \mu \mathrm{g} / \mathrm{mL}$ ) of DOX for $24 \mathrm{~h}$. Flow cytometry analysis was conducted to evaluate apoptosis of the transfected LoVo/DOX (E and G) and HCT116/DOX (F and H) cells after administrated with $2 \mu \mathrm{g} / \mathrm{mL}$ DOX for $24 \mathrm{~h}$. $* \mathrm{P}<0.05$.

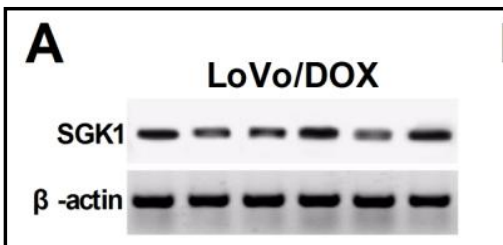

B
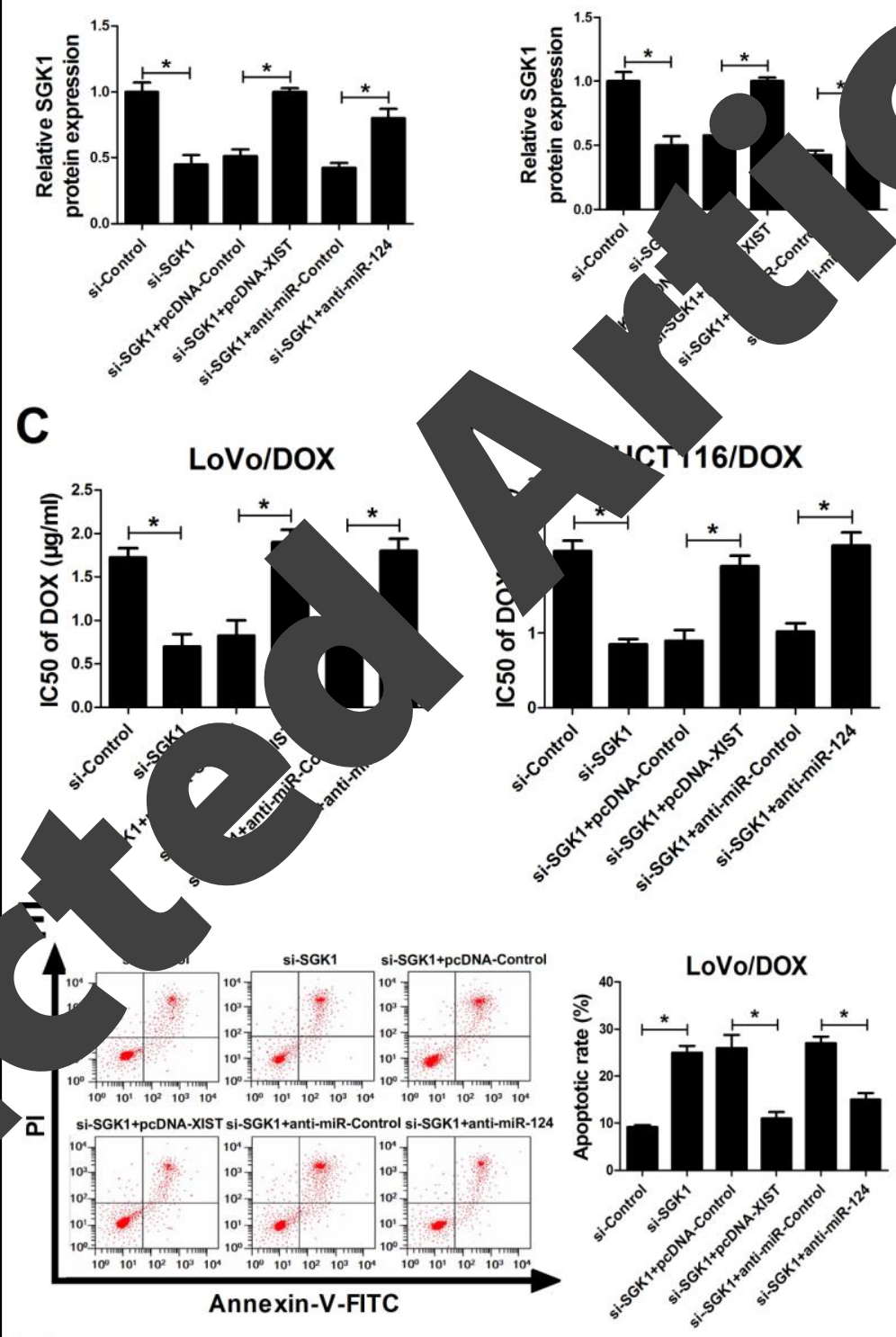

$\mathbf{F}$
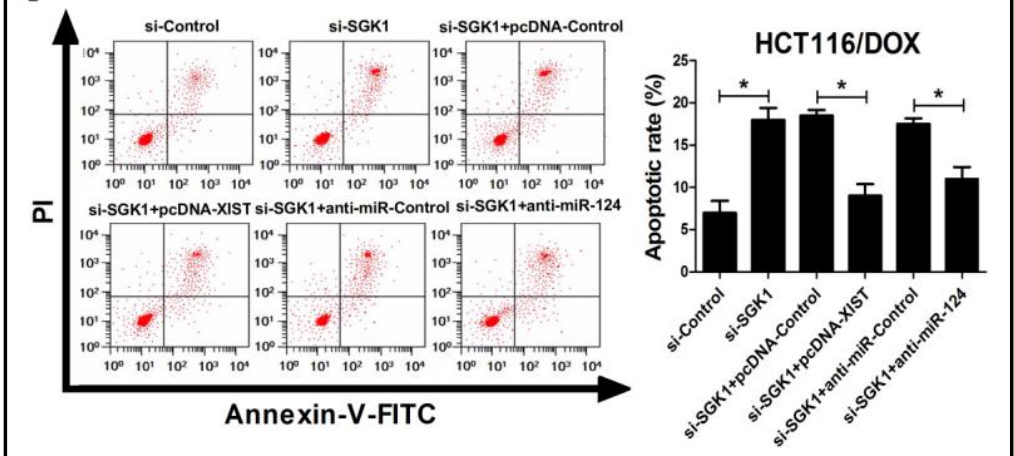


\section{\begin{tabular}{lll} 
Cellular Physiology & \multicolumn{1}{c}{ Cell Physiol Biochem 2018;51:113-128 } \\
DOl: 10.1159/000495168 & ( 2018 The Author(s). Published by S. Karger AG, Basel
\end{tabular}

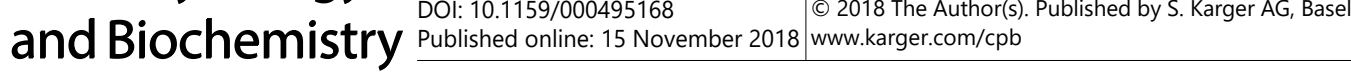 \\ Zhang et al.: XIST/miR-124/SGK1 Regulates DOX Resistance in CRC}

XIST overexpression and miR-124 inhibition reversed SGK1-knockdown-mediated inhibition of DOX resistance in DOX-resistant CRC cells

To determine the effects of XIST and miR-124 on SGK1 expression and functions in DOXresistant CRC cells, LoVo/DOX and HCT116/DOX cells were transfected with si-SGK1, or combined with pcDNA-XISTor anti-miR-124. Western blot analysis demonstrated that SGY silencing dramatically reduced SGK1 level in LoVo/DOX (Fig. 6A) and HCT116/DOX (Fis 6B) cells, while XIST overexpression or miR-124 suppression remarkably overturned siSGK1-induced decrease of SGK1 level. MTT assay indicated that interfering of SGK1 in Lo DOX (Fig. 6C) and HCT116/DOX (Fig. 6D) cells led to a substantial decline of 550 val DOX, but this effect was partially abated by enhanced XIST expression and ured 124 expression. Flow cytometry analysis confirmed that the apoptotic rate was increased following transfection with si-SGK1 in LoVo/DOX (Fig. 6E) and $\mathrm{H}^{1 / 6 / 4}$ Fig. 6F) cells, but this effect was distinctively counteracted by cotransfection w anti-miR-124. Taken together, these results revealed that SGK1 knockd resistance in DOX-resistant CRC cells, which was mediated by expresoi altera if XIST or miR-124.

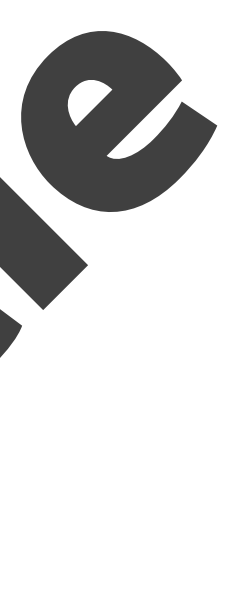

A

B
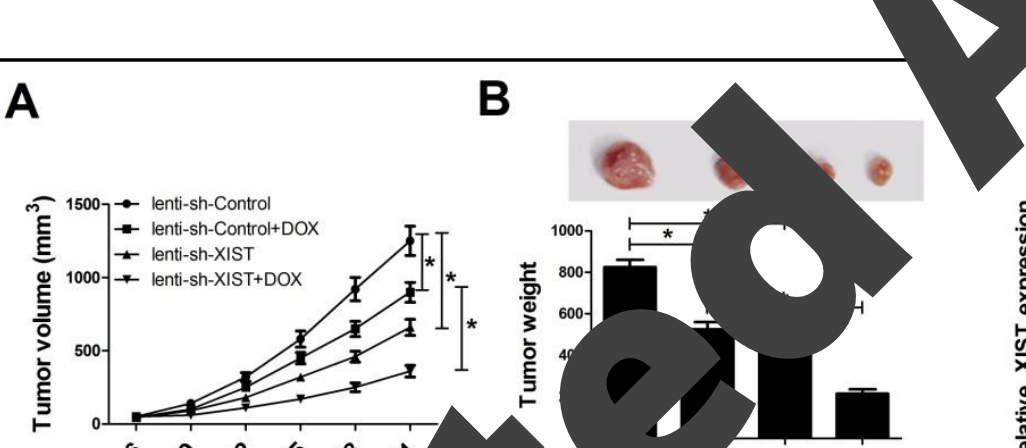

$$
\text { (1) }
$$
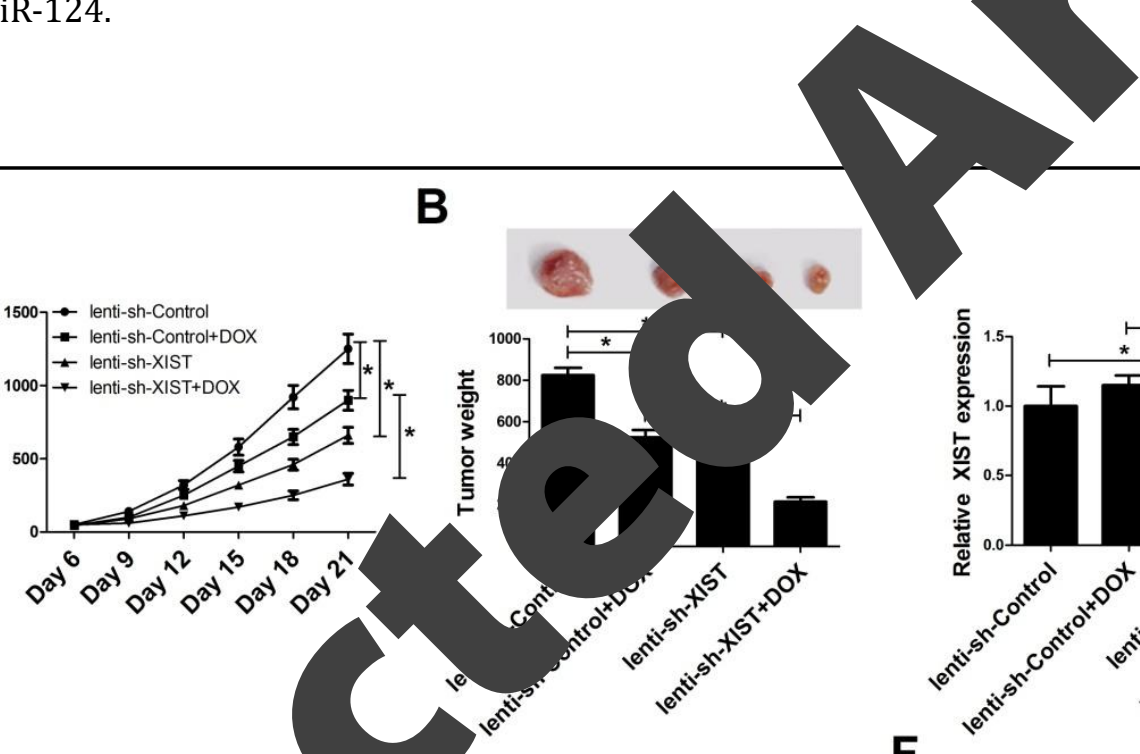

D
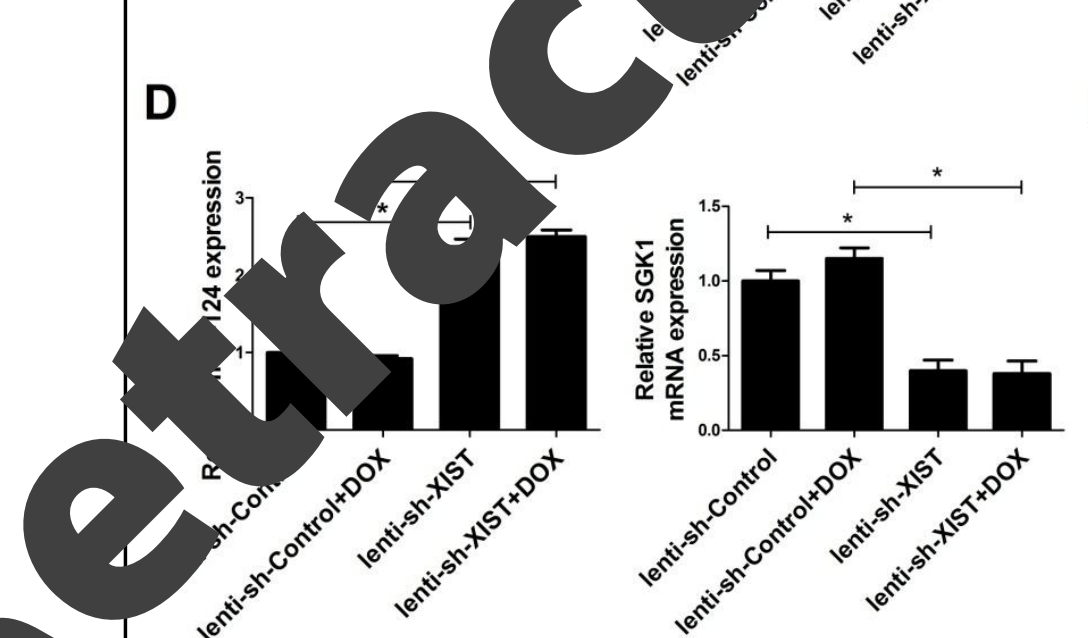

$\mathbf{F}$

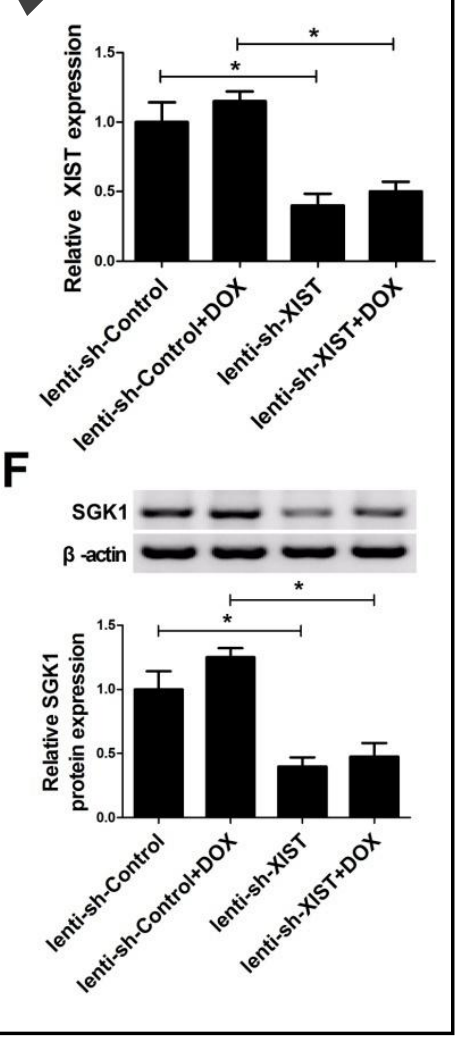

Fig. 7. XIST knockdown enhanced the anti-tumor effect of DOX in CRC in vivo. LoVo/DOX cells stably infected lenti-sh-XIST or lenti-sh-Control were subcutaneously injected into each mouse, followed by administration with DOX (5 mg/kg) every 3 days from the $6^{\text {th }}$ day after cell inoculation. (A) Tumor volumes were examined every 3 days for 21 days. (B) Mice were euthanized by cervical dislocation on day 21 after implantation, and then tumors were dissected and weighed. The expressions of XIST (C) and miR-124 (D), as well as SGK1 mRNA (E) and protein (F) levels in excised tumor masses were detected by qRT-PCR and western blot. ${ }^{*} \mathrm{P}<0.05$. 


\section{Cellular Physiology and Biochemistry \begin{tabular}{l|l} 
DOI: 10.1159/000495168 2018 The Author(s). Published by S. Karger AG, Basel \\
Publisneg
\end{tabular}

\section{XIST knockdown enhanced the anti-tumor effect of DOX in CRC in vivo}

To confirm the effects of XIST on the chemosensitivity of CRC cells to DOX in vivo, LoVo/ DOX cells stably infected with lenti-sh-XIST or lenti-sh-Control were subcutaneously injected into each mouse, followed by administration with DOX. As shown in Fig. 7A and 7B, XIST depletion significantly reinforced DOX-induced suppression on the tumor growth, present as decreased tumor volume and lowered tumor weight. qRT-PCR analysis clarified that tumo xenografts derived from sh-XIST-transfected LoVo/DOX cells showed a marked reduction XIST expression (Fig. 7C) and a remarkable increase of miR-124 expression (Fig. 7D) y or without DOX treatment. Moreover, the mRNA expression of SGK1 was coincidad well the protein levels, which illustrated a dramatic decline in the sh-XIST treatmer presence or absence of DOX (Fig. 7E and 7F). Collectively, these results implicate knockdown displayed a synergic effect with DOX in suppressing CRC cell

\section{Discussion}

Drug resistance is a frequent cause of chemotherapy are in Ch [22]. It has been proposed that numerous IncRNAs play vital fu resistance of CRC cells. For instance, knockdown of pr te transcript 1 (PCAT-1) inhibited c-Myc-dependent aggressive in CRC cells [23]. Overexpression of maternally ressed gen for CRC, promoted chemosensitivity by enhan H19 conferred methotrexate resistance throug Increasing studies revealed the oncogenic fun cancer [26], nasopharyngeal carcinoma [27] inducing cell proliferation, invasion and the carcinogenicity of XIST in CRC $[1$ XIST was significantly upregulated i. XIST inhibited 5FU-induced whether XIST could also affe. initially detected the expr cron DOX-resistant CRC tissues and cells, and found that XIST expression w a berran a pregulated in DOX-resistant CRC tissues and cells. Moreover, functional a ves Memonstrated that knockdown of XIST inhibited DOX resistance of CRC colls, as ed by the reduced IC50 value of DOX, decreased P-gp and GST- $\pi$ protein 1 enrranced apoptosis in XIST-silenced DOX-resistant CRC cells.

circuitry, of the overall activity and functional balance of gene network in a cell. An arba this system may trigger a series of pathological processes in different b^un ves, including cancers [29]. XIST inhibited cell proliferation and mobility in arco, interacting with miR-21-5p and upregulating programmed cell death 4 1) exp sssion [30]. Knockdown of XIST exerted tumor-suppressive effects in gastric and ting as a molecular sponge of miR-101 to modulate enhancer of zeste homolog EZH $z$, expression [31]. XIST overexpression enhanced cell growth in nasopharyngeal ar soma through upregulating E2F3 in part through sponging miR-34a-5p [27]. In our $y$, luciferase reporter assay and qRT-PCR analysis revealed that XIST suppressed miR4 expression by functioning as a molecular sponge in DOX-resistant CRC cells. miR-124 is a well-recognized tumor suppressor and is epigenetically silenced in various types of cancer including CRC $[16,32]$. Additionally, it was reported that restoration of miR-124 repressed Frizzled (FZD) 5 to overcome P-gp-mediated multidrug resistance in renal cell carcinoma [33]. Moreover, miR-124 was found to be downregulated in gefitinib-resistant non-small cell lung cancer (NSCLC) cells, and overexpression of miR-124 sensitized gefitinib-resistant cells to gefitinib [34]. Also, miR-124 was found to be a potential therapeutic agent to improve the efficacy of chemotherapy with DNA-damaging agents in breast cancer [35]. Our study demonstrated that miR-124 expression was downregulated in DOX-resistant CRC tissues 


\section{Cellular Physiology and Biochemistry \begin{tabular}{l|l} 
DOI: 10.1159/000495168 & $\begin{array}{l}\text { ㄷ } 2018 \text { The Author(s). Published by S. Karger AG, Basel } \\
\text { www.karger.com/cpb }\end{array}$
\end{tabular}

and cells. Ectopic expression of miR-124 enhanced DOX cytotoxicity in DOX-resistant CRC cells. Moreover, we found that suppression of miR-124 strikingly reversed XIST knockdownmediated repression on DOX resistance in DOX-resistant CRC cells.

SGK1, belonging to the AGC family of serine/threonine protein kinases, has been suggested to be overexpressed and associated with cell proliferation, apoptosis, invasivene motility, epithelial to mesenchymal transition, adhesiveness and chemoresistance in variety of epithelial tumors $[20,36]$. Amato et al. firstly revealed the association of SGK1 and doxorubicin-induced apoptosis in kidney cancer cells [37]. Moreover, a previous rep elucidated that SGK1 was highly expressed in CRC tumor tissues, and SGK1 doynregul impeded cell proliferation, migration and increased apoptosis induced by (5-FU) [38]. In this study, SGK1 was identified as a target of miR-124 in DOX-re cells. Moreover, XIST positively regulated SGK1 expression by interacti in DOX-resistant CRC cells. SGK1 expression was increased in DOX-resis and cells. Furthermore, SGK1 knockdown alleviated DOX resistance a induced apoptosis in DOX-resistant CRC cells, while these effects wera: XIST overexpression or miR-124 inhibition. In vivo experiments als firmed that XIST knockdown enhanced the anti-tumor effect of DOXon CR vivo pos vodulating miR-124 and SGK1 expressions. Together, all these results that k kdown of XIST inhibited DOX resistance in CRC by upregulation of miR-12

\section{Conclusion}

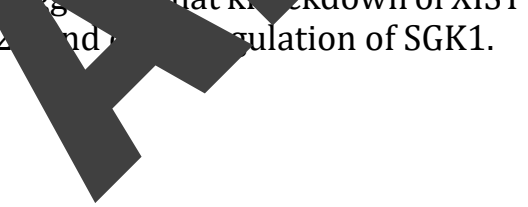

In the present study, we demonstrated th (IS) ession was upregulated and miR124 expression was downregulated in DOX-r ant tissues and cells. In addition, we provided the evidence that knockdown d resistance to DOX by working as a molecular sponge of miR-124 and sub tur reaucing SGK1 protein level in DOX-resistant CRC cells. Therefore, XIST might be a erapeutic target for improving the efficacy of DOX-based chemotherapy

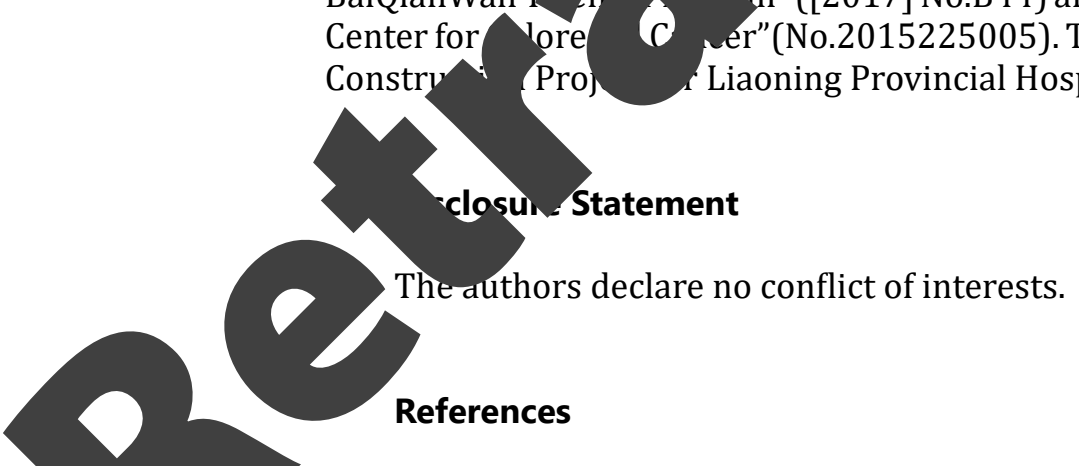

Natural Science wap sup the National Natural Science Fund from the National BaiQianWan T "m" ([2017] No.B44) and the project of "Liaoning Clinical Research Center for lore C ver"(No.2015225005). This work was granted by Clinical Capability Pro) Liaoning Provincial Hospitals (No.LNCCC-D44-2015).

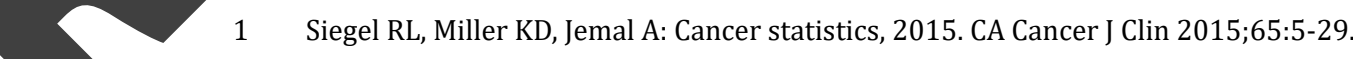

-2 Torre LA, Siegel RL, Ward EM, Jemal A: Global Cancer Incidence and Mortality Rates and Trends--An Update. Cancer Epidemiol Biomarkers Prev 2016;25:16-27.

-3 Carvalho C, Santos RX, Cardoso S, Correia S, Oliveira PJ, Santos MS, Moreira PI: Doxorubicin: the good, the bad and the ugly effect. Current Med Chem 2009;16:3267-3285.

4 Effenberger-Neidnicht K, Schobert R: Combinatorial effects of thymoquinone on the anti-cancer activity of doxorubicin. Cancer Chemother Pharmacol 2011; 67:867-874. 


\section{Cellular Physiology Cell Physiol Biochem 2018;51:113-128 \begin{tabular}{l|l|l|l|l}
\hline DOI: 10.1159/000495168 & (c) 2018 The Author(s). Published by S. Karger AG, Basel
\end{tabular}

5 Gibb EA, Brown CJ, Lam WL: The functional role of long non-coding RNA in human carcinomas. Mol Cancer 2011;10:38.

6 Kondo Y, Shinjo K, Katsushima K: Long non-coding RNAs as an epigenetic regulator in human cancers. Cancer Sci 2017;108:1927-1933.

-7 Xia H, Hui KM: Mechanism of cancer drug resistance and the involvement of noncoding RNAs. Curr Med Chem 2014;21:3029-3041.

-8 Briggs SF, Reijo Pera RA: X chromosome inactivation: recent advances and a look forward. Curr Opin Genet Dev 2014;28:78-82.

-9 Zhu J, Kong F, Xing L, Jin Z, Li Z: Prognostic and clinicopathological value of long noncoding RNA XIST cancer. Clin Chim Acta 2018;479:43-47.

10 Song H, He P, Shao T, Li Y, Li J, Zhang Y: Long non-coding RNA XIST functions as an oncogene in colorectal cancer by targeting miR-132-3p. J BUON 2017;22:696-703.

$\checkmark 11$ Chen DL, Chen LZ, Lu YX, Zhang DS, Zeng ZL, Pan ZZ, Huang P, Wang FH, Li YH, Ju HQ, noncoding RNA XIST expedites metastasis and modulates epithelial-mesenchymal t cancer. Cell Death Dis 2017;8:e3011.

12 Xiao Y, Yurievich UA, Yosypovych SV: Long noncoding RNA XIST is a prognostic and inhibits 5-fluorouracil-induced cell cytotoxicity through prom Oncotarget 2017;8:83171-83182.

13 Ha M, Kim VN: Regulation of microRNA biogenesis. Nat Rev Mol Ce

14 Magee P, Shi L, Garofalo M: Role of microRNAs in chemoresistance. A

15 Ju J: Implications of miRNAs in Colorectal Cancer C

16 Zhou L, Xu Z, Ren X, Chen K, Xin S: MicroRNA-124 (M Invasion in Colorectal Cancer by Downregulating Rho Biochem 2016;38:1785-1795.

17 Yoon JH, Abdelmohsen K, Gorospe M: Functional in Semin Cell Dev Biol 2014;34:9-14. Xiong Y, Wang L, Li Y, Chen M, He W, Qi Modulate Bladder Cancer Growth, Invas Physiol Biochem 2017;43:40,

19 Longley DB, Johnston PG: Mols

Pia X, Tang Y, Zhao Z, Zhang J, Liu H: Knockdown of long non-coding RNA prostate cancer-associated RNA vipt 1 inhibits multidrug resistance and c-Myc-dependent aggressiveness in colorectal cancer o-2 ana $1-29$ cells. Mol Cell Biochem 2018;441:99-108. colorectal cancer through activating Wnt/beta-catenin pathway. Exp Cell Res 2017;350:312-317.

26 Ma L, Zhou Y, Luo X, Gao H, Deng X, Jiang Y: Long non-coding RNA XIST promotes cell growth and invasion through regulating miR-497/MACC1 axis in gastric cancer. Oncotarget 2017;8:4125-4135.

27 Song P, Ye LF, Zhang C, Peng T, Zhou XH: Long non-coding RNA XIST exerts oncogenic functions in human nasopharyngeal carcinoma by targeting miR-34a-5p. Gene 2016;592:8-14.

28 Chang S, Chen B, Wang X, Wu K, Sun Y: Long non-coding RNA XIST regulates PTEN expression by sponging miR-181a and promotes hepatocellular carcinoma progression. BMC Cancer 2017;17:248.

29 Ergun S, Oztuzcu S: Oncocers: ceRNA-mediated cross-talk by sponging miRNAs in oncogenic pathways. Tumour Biol 2015;36:3129-3136. 


\section{Cellular Physiology Cell Physiol Biochem 2018;51:113-128

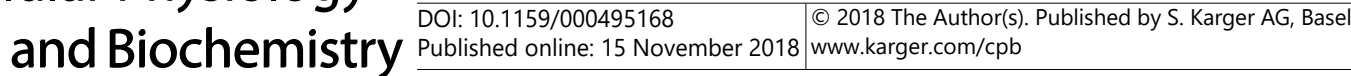 \\ Zhang et al.: XIST/miR-124/SGK1 Regulates DOX Resistance in CRC}

-30 Zhang R, Xia T: Long non-coding RNA XIST regulates PDCD4 expression by interacting with miR-21-5p and inhibits osteosarcoma cell growth and metastasis. Int J Oncol 2017;51:1460-1470.

-31 Chen DL, Ju HQ Lu YX, Chen LZ, Zeng ZL, Zhang DS, Luo HY, Wang F, Qiu MZ, Wang DS, Xu DZ, Zhou ZW, Pelicano H, Huang P, Xie D, Wang FH, Li YH, Xu RH: Long non-coding RNA XIST regulates gastric cancer progression by acting as a molecular sponge of miR-101 to modulate EZH2 expression. J Exp Clin Cancer Res 2016;35:142.

-32 Pierson J, Hostager B, Fan R, Vibhakar R: Regulation of cyclin dependent kinase 6 by microRNA 124 in medulloblastoma. J Neurooncol 2008;90:1-7.

-33 Long QZ, Du YF, Liu XG, Li X, He DL: miR-124 represses FZD5 to attenuate P-glycoprotein-mediated ch resistance in renal cell carcinoma. Tumour Biol 2015;36:7017-7026.

34 Hu FY, Cao XN, Xu QZ, Deng Y, Lai SY, Ma J, Hu JB: miR-124 modulates gefitinib resistance thro and STAT3 in non-small cell lung cancer. J Huazhong Univ Sci Technolog Med Sci 2016

-35 Chen SM, Chou WC, Hu LY, Hsiung CN, Chu HW, Huang YL, Hsu HM, Yu JC, Shen CY: The MicroRNA-124 Overexpression on Anti-Tumor Drug Sensitivity. Plos One 2015;10: $e^{n}$

-36 Lang F, Perrotti N, Stournaras C: Colorectal carcinoma cells--regulation of survival J Biochem Cell Biol 2010;42:1571-1575.

37 Amato R, Menniti M, Agosti V, Boito R, Costa N, Bond HM, Barbieri Y liaferri P, Serrotti N: IL-2 signals through Sgk1 and inhibits proliferation and apoptos 2007;85:707-721.

-38 Liang X, Lan C, Jiao G, Fu W, Long X, An Y, Wang K, Zhou J, Chen T, Li Liang X, Lan C, Jiao G, Fu W, Long X, An Y, Wang K, Zhou J, Chen T, Li
Therapeutic inhibition of SGK1 suppresses colorect

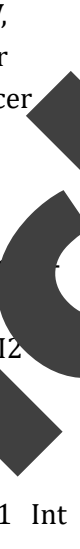

